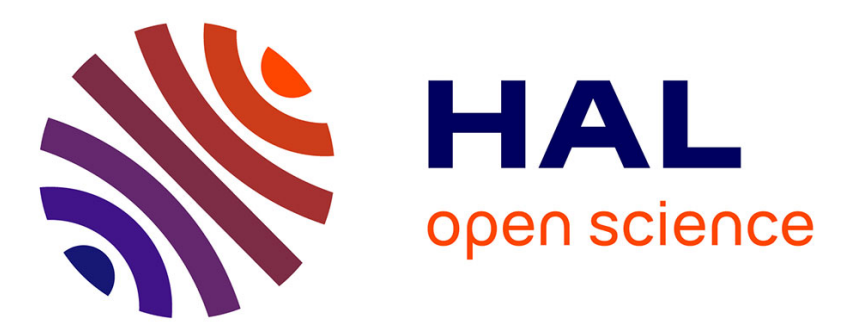

\title{
Contactless Characterization of Coplanar Stripline Discontinuities by RCS Measurement
}

Olivier Rance, Romain Siragusa, Pierre Lemaitre-Auger, Etienne Perret

\section{To cite this version:}

Olivier Rance, Romain Siragusa, Pierre Lemaitre-Auger, Etienne Perret. Contactless Characterization of Coplanar Stripline Discontinuities by RCS Measurement. IEEE Transactions on Antennas and Propagation, 2017, 65 (1), pp.251-257. 10.1109/TAP.2016.2618480 . hal-02065468

\section{HAL Id: hal-02065468 https://hal.science/hal-02065468}

Submitted on 12 May 2020

HAL is a multi-disciplinary open access archive for the deposit and dissemination of scientific research documents, whether they are published or not. The documents may come from teaching and research institutions in France or abroad, or from public or private research centers.
L'archive ouverte pluridisciplinaire HAL, est destinée au dépôt et à la diffusion de documents scientifiques de niveau recherche, publiés ou non, émanant des établissements d'enseignement et de recherche français ou étrangers, des laboratoires publics ou privés. 


\title{
Contactless Characterization of Coplanar Stripline Discontinuities by RCS Measurement
}

\author{
O. Rance, R. Siragusa, P. Lemaître-Auger, and E. Perret, Senior Member IEEE
}

\begin{abstract}
The article presents a contactless approach to characterize coplanar stripline discontinuities based on a radar cross-section (RCS) measurement method. With this approach, the values of the equivalent lumped element models are determined for two discontinuities often encountered in practice: open-circuit and short-circuit. The discontinuities are incorporated into two different very simple resonators. But contrarily to what is usually done, here the resonators are considered as radar resonant targets and are illuminated by a plane wave. The incremental electrical length due to the discontinuities causes a shift of the resonance frequency which is used for the extraction of the parameters. The values obtained from full-wave simulations are compared to measurements and to other data available from previous studies.
\end{abstract}

Index Terms-Coplanar stripline discontinuities, RCS measurement, resonator, wireless method.

\section{INTRODUCTION}

A COPLANAR stripline (CPS) is a uniplanar transmission line (TL) whose structure consists in two conductor strips separated by a gap and placed on a dielectric substrate of finite thickness $D$ [Fig. 1(a)]. This configuration corresponds to the dual structure of the coplanar waveguide (CPW) and provides the same advantages, including small losses, easy mounting of series lumped component and no need for via due to the uniplanar configuration.

CPSs are commonly used in monolithic microwave integrated circuits [1], as well as in microwave components such as filters [2]. Lately, CPSs have been used in various application fields such as antennas [3], chipless radio frequency identification (RFID) [4], or for medical sensors [5].

Compared to the extensive work carried out on microstrip and CPW structures, only few studies have been made to obtain lumped equivalent circuits of CPS discontinuities. The first characterization of simple CPS discontinuities has been reported in [6]. The values of the model elements were extracted from simulations (finite-difference time domain (FDTD) method) and measurements by using, in both cases, a thru-reflect-line (TRL) calibration algorithm. Some limitations concerning the de-embedding procedure of [6] are highlighted in [7] such as the parasitic effects of the calibration standards which are ignored. To correct that, $\mathrm{Zhu}$ and $\mathrm{Wu}$ present in [7]

O. Rance, P. Lemaître-Auger, R. Siragusa, E. Perret are with the Laboratoire de Conception et d'Intégration des Systèmes (LCIS) - Grenoble Institute of Technology, Valence, France (e-mail: olivier.rance@lcis.grenoble-inp.fr; pierre.lemaitre-auger@lcis.grenoble-inp.fr romain.siragusa@lcis.grenobleinp.fr; etienne.perret@lcis.grenoble-inp.fr). E. Perret is also with the Institut Universitaire de France, Paris, France.

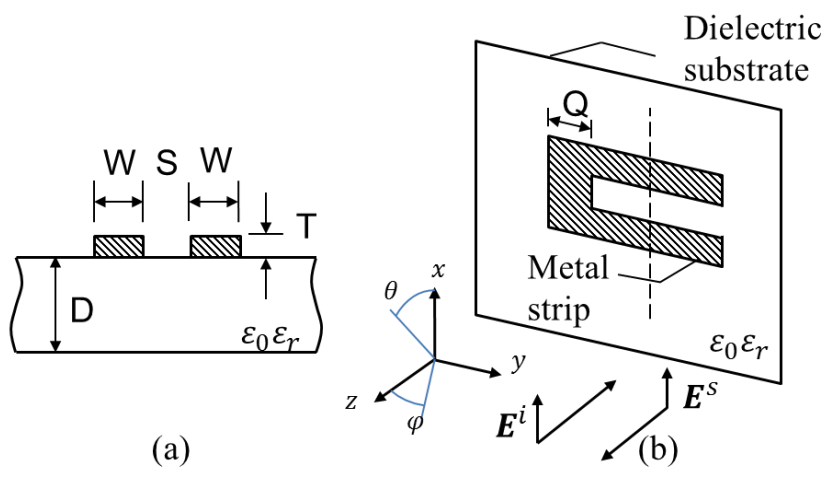

Fig. 1. (a) Transverse section of coplanar stripline (CPS). (b) RCS measurement principle. A resonator that incorporates the discontinuity to be tested is illuminated by a plane wave $\boldsymbol{E}^{i}$. The backscattered field $\boldsymbol{E}^{S}$ is measured.

a second characterization method based on a deterministic method of moment (MoM) combined with a short-open calibration (SOC) technique for a more accurate assessment of the parameter values. Up to now, those two methods ([6], [7]) are the only ones existing for an experimental characterization of CPS discontinuities. They are both based on guided approaches that need an accurate de-embedding procedure.

Approximate analytical approaches were also proposed for a theoretical evaluation of open and short circuit discontinuities. A general quasi-TEM model is proposed in [8] for two wire transmission lines. It yields simple closed-form expressions that can be applied to the case of CPS. Another work presents a computation method based on the quasi-static concept of excess charge solved by MoM [9].

This article presents a novel experimental approach to characterize short circuit (SC) and an open circuit (OC) discontinuities of CPS based on RCS measurements. Compared to previous approaches, it does not need any deembedding techniques or complex feeding network [7]. Indeed, this method simply uses a resonant scatterer that incorporates the discontinuity to be tested. The shift of the resonance frequency due to the discontinuity is then used to extract the values of the lumped equivalent elements. We note that this method belongs to the more general "resonator approach" used to analyze the microstrip discontinuities [1], [10], [11]. But contrarily to what is classically done with microstrip, here, the resonator does not need to be coupled to a feed line through a gap. The resonator is considered as a radar target which is illuminated by a plane wave [Fig. 1(b)]. This way of doing eliminates the possible errors due to the gap perturbation and the de-embedding techniques. Similar RCS measurement method have been used for the characterization 
of antennas with small electric length, scatterers, or chipless RFID tags [12]-[15].

The characterization method base on the resonator approach is presented in detail in section II. Section III is dedicated to the RCS measurement approach. Simulation and measurement results are compared to each other and also with published data.

\section{CHARACTERIZATION METHOD}

\section{A. Discontinuity Models}

The discontinuities under study are the SC and the OC.

First, a CPS short circuit is realized by interconnecting the strips with a conductor at the plane $P_{l}$ as it is shown in Fig. 2(a). At radiofrequency, magnetic energy is stored behind the termination so that the structure departs from an ideal SC. This can be modeled either by a lumped inductance $L_{s}$ or by an incremental length $\Delta l_{s}$ of the strips terminated with and ideal SC. This double modeling of the SC is the basic idea of the proposed method. Indeed, $L_{S}$ is the element which will be experimentally evaluated while the extra length, $\Delta l_{s}$, is the element that will be detected through the measurement of a frequency shift, like it will be seen in the following section.

Obviously, the impedance seen at the plane $P_{l}$ has to be the same for both models [10]. So considering no loss:

$$
\omega L_{s}=Z_{0} \tan \left(\beta \Delta l_{s}\right)
$$

where $\omega$ is the pulsation, and $Z_{0}$ and $\beta$ are the characteristic impedance and the wavenumber of the CPS line.

Second, a CPS OC is formed by abruptly ending the metallic strips at the plane $P_{2}$ as it can be seen in Fig. 2(b). The dominant phenomenon associated with $\mathrm{OC}$ is the apparition of fringing fields which are extending beyond the physical termination of the metallic strips [6]. This gives rise to a capacitive reactance which can be modeled either by a lumped shunt capacitance $C_{0}$ or by an incremental length $\Delta l_{o}$. Like in the previous case, those two equivalent representations are essential. With the same argument as for the SC case [10], we find:

$$
-1 / \omega C_{o}=-Z_{0} / \tan \left(\beta \Delta l_{o}\right) .
$$

A simpler expression of $L_{s}$ and $C_{0}$ is obtained using the first term of the expansion of the tangent function:

$$
\begin{aligned}
& L_{s}=Z_{0} \Delta l_{s} \sqrt{\varepsilon_{e f f}} / c, \\
& C_{0}=\Delta l_{o} \sqrt{\varepsilon_{e f f}} / c Z_{0} .
\end{aligned}
$$

where $\varepsilon_{\text {eff }}$ is the effective permittivity of the CPS line.

\section{B. Resonator Approach}

The SC and OC discontinuities are incorporated into two different resonant scatterers represented in Fig. 3. The loop resonator [Fig. 3(a)] can be considered as a TL section terminated at both ends by a SC. The C-like resonator [Fig. $3(\mathrm{~b})$ ] is a TL section terminated by a SC at one end and by an $\mathrm{OC}$ at the other end. A third kind of resonator terminated at both end by an OC could be theoretically used but it is hardly excited by a plane wave illumination and therefore it is not applicable for the present kind of measurement. For the study,

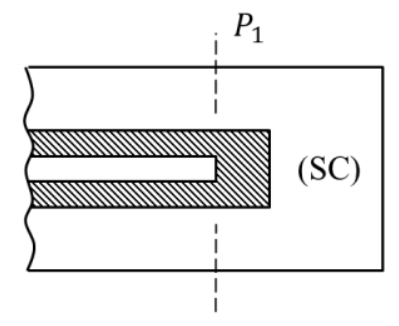

(a)
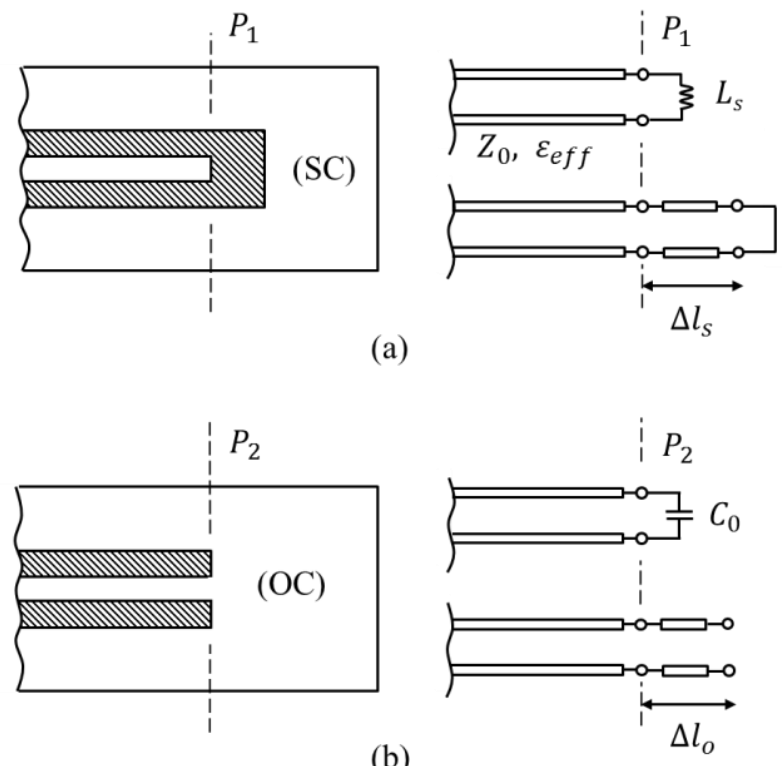

(b)

Fig. 2. Physical CPS discontinuities and their models. (a) Short circuit modeled either by a lumped inductance $L_{S}$ or by an additional TL section of length $\Delta l_{s}$. (b) Open circuit modeled either by a lumped capacitance $C_{0}$ or by an additional TL section of length $\Delta l_{0}$.

$(\mathrm{SC})$

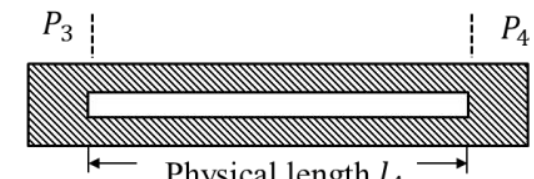

(SC)

(SC)

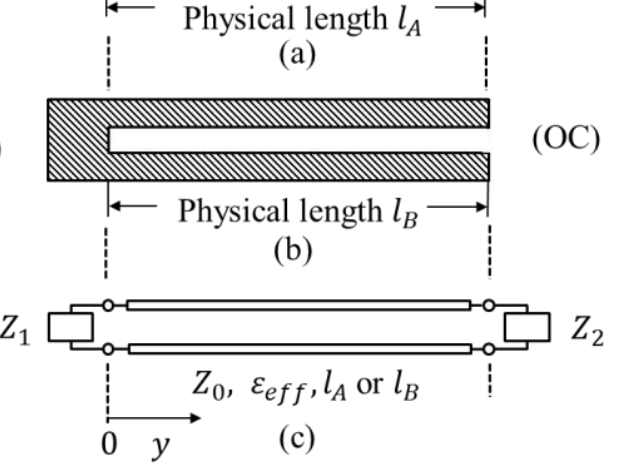

Fig. 3. Complete physical structure of the two scatterers and the equivalent circuit. (a) Loop-shaped scatterer (half-wavelength resonator). (b) C-likeshaped scatterer (quarter-wavelength resonator). (c) Equivalent resonant circuit.

the TL and the discontinuities are considered lossless. At resonance, the geometrical lengths $l_{A}$ and $l_{B}$ of the resonators (see Fig. 3) are related to the guided wavelength $\lambda_{r}$. The relation is classically obtained from the study of the resonance equation associated to the resonant circuit shown in Fig. 3(c). The detail of calculation is given in Annexe A.

The loop scatterer behaves like a half-wavelength resonator:

$$
l_{A}+2 \Delta l_{s}=m \lambda_{r} / 2,
$$

where $m$ is an integer.

The C-like scatterer behaves like a quarter-wavelength resonator:

$$
l_{B}+\Delta l_{s}+\Delta l_{o}=\lambda_{r} / 4+m \lambda_{r} / 2 .
$$

It is worth to note that in (5) and (6), the incremental lengths $\Delta l_{s}$ and $\Delta l_{o}$ (or their equivalent lumped elements $L_{s}$ 
and $C_{0}$ ) are functions of the frequency which directly depends on the physical length of the strips.

The guided wavelength is related to the resonant frequency $f_{r}$ :

$$
\lambda_{r}=c / f_{r} \sqrt{\varepsilon_{\text {eff }}}
$$

where $c$ denotes the speed of light in free space and the effective permittivity $\varepsilon_{e f f}$ is calculated from the physical dimensions of the TL using the closed form equation given in [16]. Considering the first resonance for each scatterer, we have:

$$
\begin{gathered}
l_{A}+2 \Delta l_{S}\left(f_{A}\right)=c / 2 f_{A} \sqrt{\varepsilon_{e f f}}, \\
l_{B}+\Delta l_{S}\left(f_{B}\right)+\Delta l_{o}\left(f_{B}\right)=c / 4 f_{B} \sqrt{\varepsilon_{e f f}},
\end{gathered}
$$

where the parenthesis denote the dependence on the frequency.

As it can be seen from (8)-(9), the incremental length due to the discontinuities causes a shift of the resonance frequency compared to the perfect SC and OC models. The value $\Delta l_{s}$ corresponding to the resonant frequency $f_{A}$ can be calculated from (8). If the physical length $l_{A}$ and $l_{B}$ are chosen such that the resonant frequency of both scatterers $f_{A}$ and $f_{B}$ are equal, we can replace $\Delta l_{s}$ by its expression in (9), and then calculate $\Delta l_{o}$. Considering that $\varepsilon_{e f f}$ and $Z_{0}$ are constant with frequency which is a classic approximation for quasi-TEM lines, the corresponding values of $L_{s}$ and $C_{0}$ can then be obtained from (1) and (2).

\section{RCS MEASUREMENT}

\section{A. Realized scatterers}

Two series of resonant scatterers having different geometrical dimensions have been realized for experimental validation of the characterization method. For the series $n^{\circ} 1$, the geometrical dimensions of the CPS elements are taken identical to the one of [6] for comparison with previously published data. A picture of the scatterers realized for the series $\mathrm{n}^{\circ} 1$ is given in Fig 4. Until now, only the discontinuities with dimensions given in [6] have been measured in the literature. However they are difficult to realize with good accuracy for low cost realization process which can result in poor quality prototypes, like it can be seen in the expanded view in Fig. 4(b). A second series of resonators (series $n^{\circ} 2$ ) has been designed to have geometrical dimensions simple to achieve with a chemical etching realization process which can be interesting for future measurement and comparison.

The geometrical parameters of the CPS lines are indicated in Fig. 1 and their values are indicated for both series in Table I. The dielectric substrate of the series $\mathrm{n}^{\circ} 1$ consists in RTDuroid 6010 of thickness $0.76 \mathrm{~mm}$ and permittivity $\varepsilon_{r}=10.2$. The dielectric substrate of the series $\mathrm{n}^{\circ} 2$ consists in RO3003 of thickness $0.76 \mathrm{~mm}$ and permittivity $\varepsilon_{r}=3$. The electrical parameters $\varepsilon_{e f f}$ and $Z_{0}$ in table I are calculated from the closed form equations given in [16] which are generally considered accurate for quasi-TEM lines.

The series $n^{\circ} 1$ is composed of 4 Loop scatterers and 4 C-

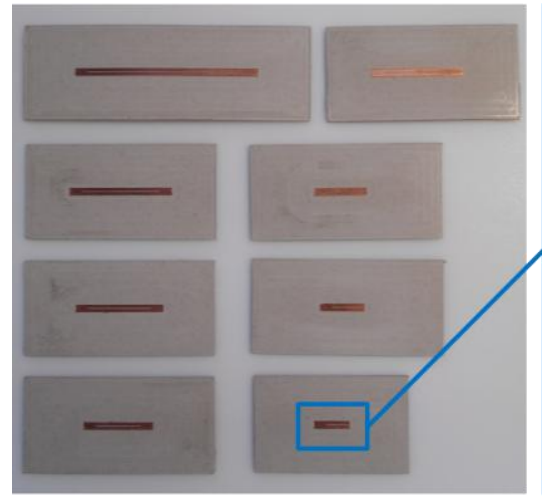

(a)

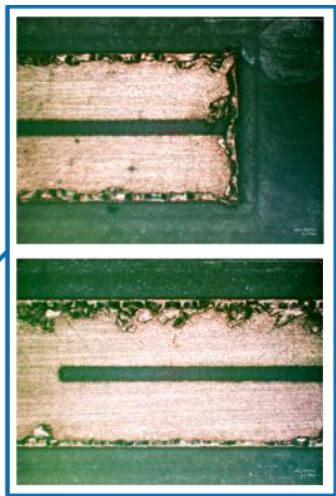

(b)
Fig. 4. Pictures of the realized scatterers of the series $n^{\circ} 1$.. (a) Pictures of the 8 realized scatterers: 4 loop and $4 \mathrm{C}$-like. For each couple of scatterer, the length of the strips is chosen such that $l_{A}=2 l_{B}$. (b) Expanded view of a Clike scatterer.

TABLE I

GEOMETRICAL DIMENSIONS OF THE REALIZED TAGS

\begin{tabular}{|c||c||c|}
\hline Parameter & Series $\mathrm{n}^{\circ} 1$ & Series $\mathrm{n}^{\circ} 2$ \\
\hline \hline$D$ & $0.762 \mathrm{~mm}$ & $0.762 \mathrm{~mm}$ \\
$W$ & $0.724 \mathrm{~mm}$ & $2 \mathrm{~mm}$ \\
$S$ & $0.140 \mathrm{~mm}$ & $0.8 \mathrm{~mm}$ \\
$T$ & $17.5 \mu \mathrm{m}$ & $17.5 \mu \mathrm{m}$ \\
$Q$ & $0.254 \mathrm{~mm}$ & $5 \mathrm{~mm}$ \\
$\varepsilon_{r}$ & 10.2 & 3 \\
$\varepsilon_{\text {eff }}$ & 5.15 & 1.6 \\
$\mathrm{Z}_{0}$ & $67.5 \Omega$ & $149.7 \Omega$ \\
\hline
\end{tabular}

TABLE II

STRIP LENGTH OF THE REALIZED SCATTERERS

\begin{tabular}{|c|c|c|c|c|c|c|}
\hline \multicolumn{7}{|c|}{ Series $^{\circ} 1$} \\
\hline $\begin{array}{c}\text { Resonant } \\
\text { frequency }(\mathrm{GHz})\end{array}$ & 2 & 4 & 5 & 7 & & \\
Loop: $l_{A}(\mathrm{~mm})$ & 33.0 & 16.5 & 13.2 & 9.4 & - \\
C-like: $l_{B}(\mathrm{~mm})$ & 16.5 & 8.2 & 6.6 & 4.7 & & \\
\hline \hline \multicolumn{7}{|c|}{ Series $\mathrm{n}^{\circ} 2$} \\
\hline $\begin{array}{c}\text { Resonant } \\
\text { frequency }(\mathrm{GHz})\end{array}$ & 2.5 & 3 & 3.5 & 4 & 4.5 & 5 \\
Loop: $l_{A}(\mathrm{~mm})$ & 45.3 & 37.4 & 31.8 & 27.6 & 24.4 & 21.8 \\
C-like: $l_{B}(\mathrm{~mm})$ & 21.8 & 17.8 & 15.0 & 12.9 & 11.3 & 10.1 \\
\hline
\end{tabular}

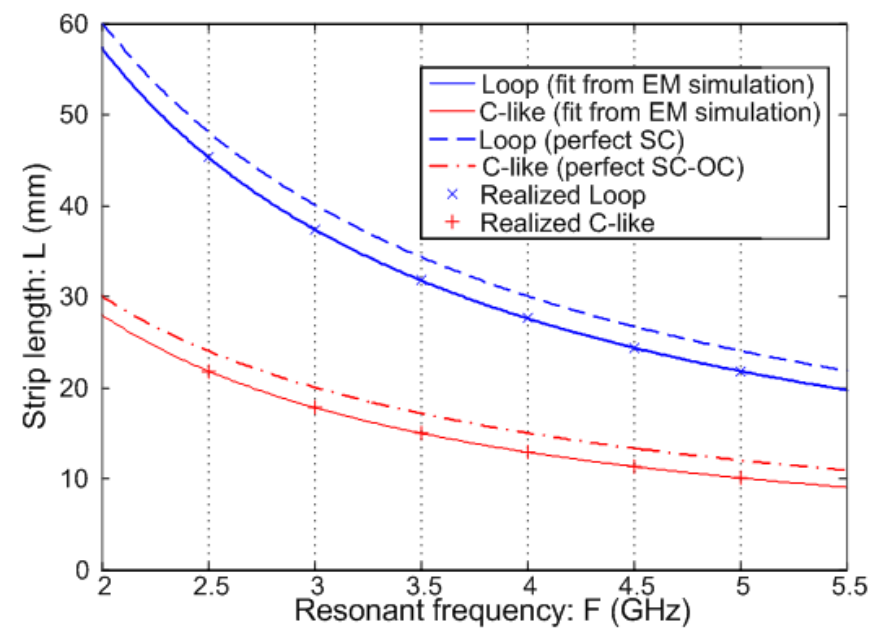

Fig. 5. Strip length in function of the resonant frequency obtained from a series of fifteen simulations for each type of scatterer. The curve is compared to perfect SC and OC circuit models. Six couples of scatterer resonating at the same frequencies have been determined from this parameter study. 
like scatterers. The length of the strips has been calculated assuming that the discontinuities behave like perfect SC or OC. The length of the strips and their corresponding approximate resonant frequencies are indicated in Table II. Here, for each couple, the length of the strips are such that $l_{A}=2 \cdot l_{B}$. As a consequence, $f_{A}$ is not exactly equal to $f_{B}$ but close enough to it. It is thus assumed that $\Delta l_{S}\left(f_{A}\right) \approx \Delta l_{S}\left(f_{B}\right)$. Solving (9) thus gives an approximate value $\Delta l_{0}{ }^{\prime}\left(f_{B}\right)$. It is easy to show from (8)-(9) that the error on $\Delta l_{o}$ can be expressed as:

$$
\Delta l_{0}^{\prime}\left(f_{B}\right)-\Delta l_{0}\left(f_{B}\right)=\Delta l_{s}\left(f_{B}\right)-\Delta l_{s}\left(f_{A}\right) .
$$

From simulations, we have observed that the approximate approach gives a maximum error of $3 \%$ on $\Delta l_{o}$ compared to the rigorous method for the frequency range of interest.

The series $n^{\circ} 2$ is composed of 6 Loop scatterers and $6 \mathrm{C}$ like scatterers. The lengths of the resonators have been chosen to obtain exactly $f_{A}=f_{B}$. Fifteen EM simulations have been performed for each resonator to determine the curves of the resonance frequency in function of the physical length presented Fig. 5. The simulation results are compared to the perfect SC and OC models. Six couples of scatterers having the same resonance frequencies have been determined based on this parametric study. The resonance frequencies and associated lengths of the realized scatterers are indicated in table II.

The simulations have been performed using the time domain solver of the commercial full-wave simulator CSTMicrowave-Studio 2016. A plane wave port is placed at a distance $d=1 \mathrm{~m}$, from the resonator and a pulse of $1 \mathrm{~W}$ is delivered in the frequency band $0 \mathrm{GHz}-20 \mathrm{GHz}$. A typical RCS level of $-30 \mathrm{dBsm}$ has been computed for the two scatterers at resonance for both series. It can be seen from simulations that the re-radiation pattern of the scatterers is nearly isotropic for small values of the gap $g$. The opposite current flows arising along the strips indicate a negligible horizontal component of the backscattered E-field ( $-150 \mathrm{dBsm}$ in simulation). The re-radiation properties of the C-like scatterer have been studied in detail in [17]. There is no detailed study about the Loop used as a scatterer in the literature but simulations are showing a similar behavior for both kinds of scatterers.

\section{B. Extraction of the natural frequencies}

The extraction of the lumped element values relies on the accurate measurement of the resonance frequency of the scatterers. It is thus necessary to ensure a robust detection which does not depends on environmental factors.

The singularity expansion method (SEM) [18] is well adapted to the description of resonant target. It is based on the observation that the scattered field response of a conducting object in the late-time can be written as a sum of damped sinusoids like:

$$
r(t)=\sum_{i=1}^{N} a_{n} e^{\sigma_{n} t} \cos \left(\omega_{n} t+\phi_{n}\right), \quad t>T_{L}
$$

Where $T_{L}$ is the beginning of the late time response, $a_{n}$ and $\phi_{n}$ are the aspect dependent amplitude and phase of the $n$th mode, and $s_{n}=\sigma_{n}+j \omega_{n}$ is the complex natural resonance associated to the $n$th mode. In (11), only $N$ resonant modes are assumed excited by the incident field waveform. A major finding of the SEM theory is that the natural resonances are not dependent on excitation or aspect. The set of natural frequencies is unique to a specific target and provides an interesting basis for target identification [18]. Simple resonant scatterers like the Loop or the C-like scatterers can be assimilated to a single scattering center and the summation (11) thus reduces to a single term. In this simple case, the natural frequency corresponds to the frequencies of the first resonant mode $f_{A}$ and $f_{B}$ appearing in (8)-(9).
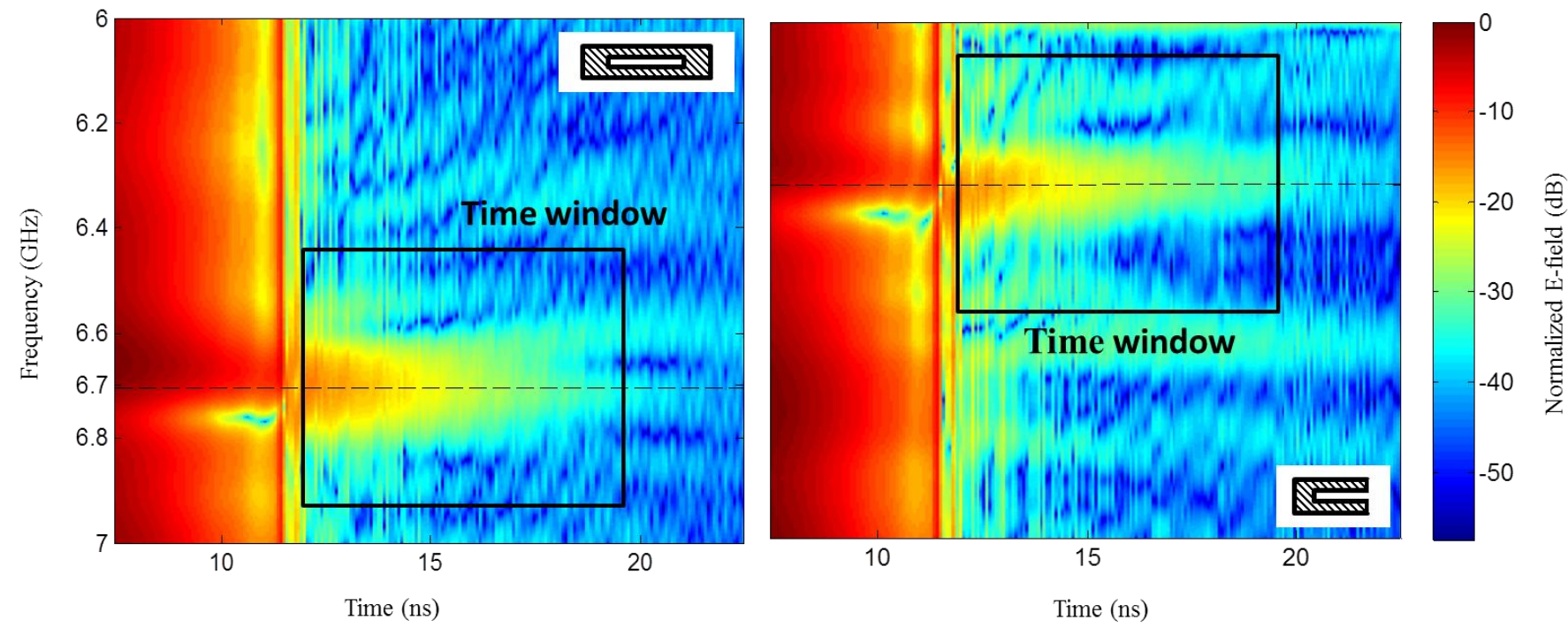

Fig. 6. Measured spectrograms of scatterers operating around $7 \mathrm{GHz}$. (a) Loop scatterer. (b) C-like scatterer. The specular reflection due to the dielectric substrate is observed for time inferior to $12 \mathrm{~ns}$ and can shift the resonant frequency of the scatterers. A time-frequency window (in black) is applied to recover the natural resonance of the scatterer. 
During the earl-time of the response, the RCS of the resonator is comparable to the one of the dielectric substrate and a shift of the resonance frequency is observed due to this additional contribution. A method to recover the natural resonance is to observe the spectrogram of the signal and to isolate the resonant part (late-time) from the specular response of the substrate (early-time) by application of a timefrequency window [19]. Measured spectrograms of the loop and the $\mathrm{C}$-like scatterers operating around $7 \mathrm{GHz}$ (series $\mathrm{n}^{\circ} 1$ ) are presented in Fig. 6. The specular reflection due to the dielectric substrate is observed in the region inferior to $12 \mathrm{~ns}$. The resonance lasts longer than the specular reflection (approximately until $20 \mathrm{~ns}$ ) and the time window is chosen to keep only the resonant part of the signal. In this manner, the natural resonance frequency of the scatterer is obtained. Application examples of this extraction method including discussion about the nature and size of the time window are given in [20].

\section{Measurement Results}

Measurements are performed in the frequency domain in an anechoic chamber, see Fig. 7, with the Agilent PNA Network Analyzer N5222A in bi-static configuration for the vertical polarization. The power delivered by the vector network analyzer is $0 \mathrm{dBm}$ in the frequency band from $2 \mathrm{GHz}$ to $8 \mathrm{GHz}$. The two horn antennas have a gain of $12 \mathrm{dBi}$ within the frequency band of interest. The spacing between the antennas is $e=30 \mathrm{~cm}$ and the distance between the tag and the antennas is $r=60 \mathrm{~cm}$. Using the coordinate system of Fig. 1, the aspect angles for the measurement are given by $\theta=90^{\circ}$ and $\varphi= \pm 15^{\circ}$. An isolation measurement and a reference measurement with a metallic plate having a known RCS were performed for calibration [21].

The natural frequencies of the realized scatterers are extracted using the procedure described in the last section. The measured resonant frequency of the Loop is used to solve (8) which determines $\Delta l_{s}$. In the same manner the resonant frequency measured for the C-like scatterers is used along with the value of $\Delta l_{s}$ in (9) to determine $\Delta l_{o}$. The corresponding lumped element values are then calculated with (1)-(2).

The measurement results obtained for the series $n^{\circ} 1$ are presented Fig. 8. The normalized reactance obtained in simulation and measurements for the SC discontinuities is compared to other results from previously published studies [6]-[9] in Fig. 8(a). A good agreement is observed between our results, quasi-static theoretical predictions and measurements. The agreement remains acceptable with the other extraction methods. A maximum relative error of $23 \%$ is obtained between measurement and theory at $2 \mathrm{GHz}$.

Fig. 8(b) similarly gives a comparison between our results and other results for the normalized reactance of the OC discontinuity. The simulation results are in excellent agreement with the quasi-TEM predictions from [8], but are more distant from the full-waves computations and measurement. The measurement curves have an irregular shape which denotes a significant level of uncertainty. A maximum relative error of $34 \%$ is obtained between measurement and theory at $4.5 \mathrm{GHz}$. The discrepancy between measurement results and theory can be explained by the poor quality of the realized prototypes, like it can be seen in Fig. 4(b). Furthermore, the derivation of $\Delta l_{o}$ depends directly on the assessment of $\Delta l_{s}$. Therefore it cumulates the inaccuracies in the realization of the two scatterers. A better agreement between simulations and measurements would certainly be obtained with a more accurate realization process. The measurement results obtained for the series $\mathrm{n}^{\circ} 2$ are compared with simulation Fig. 9 with a good agreement. The normalized reactance obtained in simulation and measurements for the $\mathrm{SC}$ are compared Fig. 9(a). A maximum relative error of $13 \%$ is obtained at $2.5 \mathrm{GHz}$. The relative error is inferior to $6 \%$ for other frequencies. Fig. 9(b) gives a comparison between

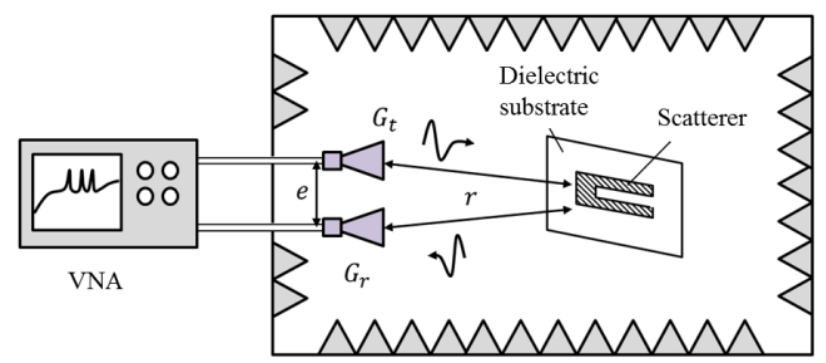

Fig. 7. Schematic representation of the measurement setup.

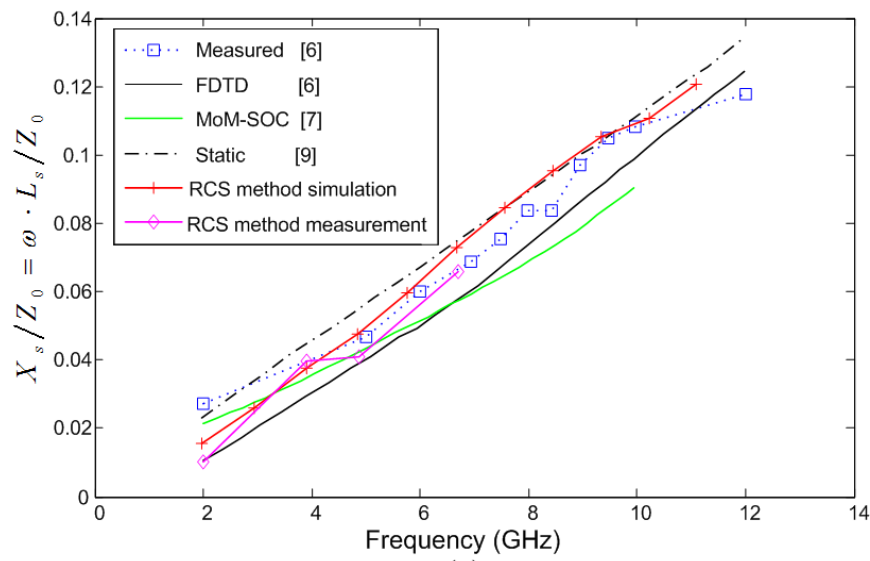

(a)

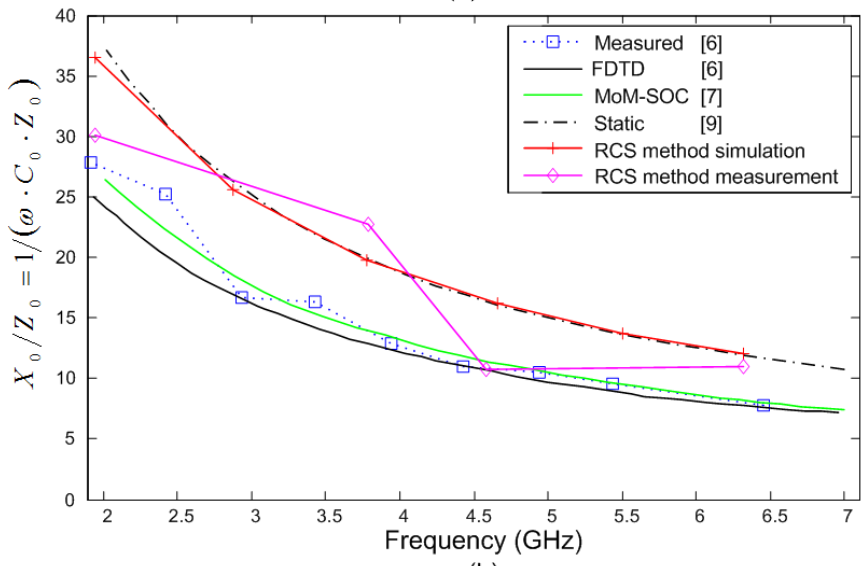

(b)

Fig. 8. Comparison of the normalized reactance of discontinuities (series $\mathrm{n}^{\circ} 1$ ) with published data. (a) Short circuit. (b) Open-circuit. 


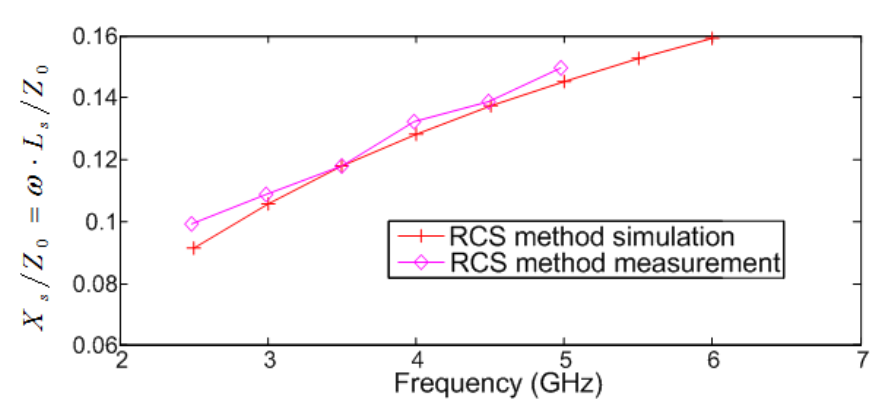

(a)

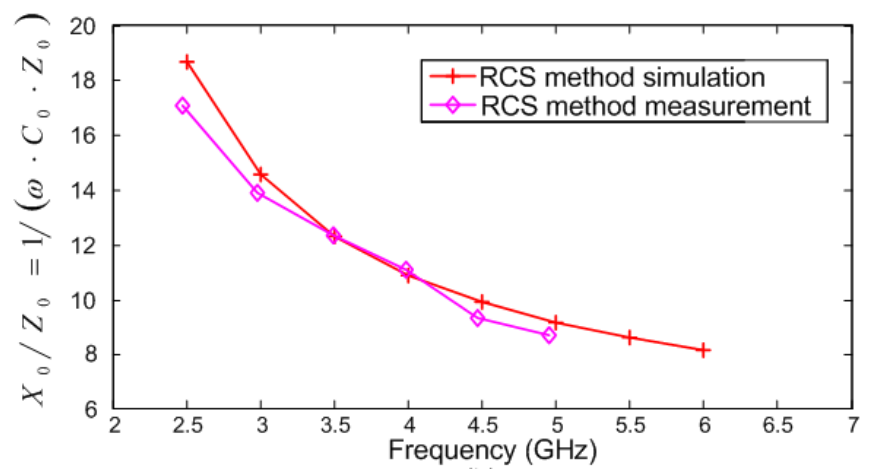

(b)

Fig. 9. Comparison of the normalized reactance of discontinuities obtained by simulation and measurement (series $n^{\circ} 2$ ). (a) Short circuit. (b) Open-circuit.

measurements and theory for the normalized reactance of the OC discontinuity. A maximum relative error of $8 \%$ is obtained at $2.5 \mathrm{GHz}$. The results are in a better agreement for series $\mathrm{n}^{\circ} 2$ which can be explained by the fact that the dimensions are simple to realize in practice. This study highlights that the extracted parameter depends strongly on the fabrication error for CPS structures. This problem also appears in [6], [7] but without particular mention.

\section{CONCLUSION}

A novel contactless method based on resonators has been proposed for the extraction of CPS discontinuities equivalent lumped elements. Contrarily to what is classically done for microstrip, the resonators are not coupled to a feed line through a gap. The discontinuities are incorporated into simple scatterers whose resonant frequencies are determined by RCS measurements. This feeding eliminates the possible errors caused by the gap uncertainties and do not require any deembedding. The measurement results have been compared with success to simulations and to available data from previous studies [6]-[9]. A new set of discontinuities has been designed with dimensions simple to realize. This technique could be easily extended to other type of discontinuities like those proposed in [6]. Furthermore, this approach does not necessarily require the use of expensive laboratory device like a VNA as it is possible to use commercial UWB radar with frequency resolutions compatible with the approach [22].

\section{APPENDIX}

Annex A: Derivation of the relation between geometrical length and guided wavelength at resonance.

The resonant frequency of the scatterer is obtained from the resonant equation:

$$
\grave{Z}+\vec{Z}=0,
$$

where $\overleftarrow{Z}$ and $\vec{Z}$ are the impedances looking at the left and right, respectively, from an arbitrary reference plane. For convenience of calculation, the reference plane is chosen at

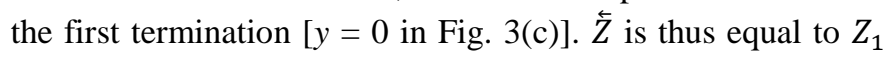
while $\vec{Z}$ is easily obtained from classical TL result, giving the following equality:

$$
Z_{1}=-\left[Z_{0} \frac{Z_{2}+j Z_{0} \tan (\beta l)}{Z_{0}+j Z_{2} \tan (\beta l)}\right]
$$

where $Z_{1}$ and $Z_{2}$ are the load impedances at both ends of the equivalent circuit, $Z_{0}$ is the characteristic impedance of the CPS, $\beta$ and $l$ are the wavenumber and the physical length of the TL as indicated in Fig. 3(c).

In the following, we consider that the terminations are only reactive:

$$
Z_{1}=j X_{1}, \quad Z_{2}=j X_{2} .
$$

where $X_{1}$ and $X_{2}$ are reactances. By solving (4) for $\tan (\beta l)$, we obtain:

$$
\tan (\beta l)=\frac{Z_{0}\left(X_{1}+X_{2}\right)}{X_{1} X_{2}-Z_{O}^{2}} .
$$

By replacing $X_{1}$ and $X_{2}$ thanks to (1) and (2), a relation between the electrical length of the scatterers to the guided wavelength at resonance $\lambda_{r}$ can be found $\left(\beta=2 \pi / \lambda_{r}\right)$

For the loop scatterer, $X_{1}=X_{2}=Z_{0} \tan \left(\beta \Delta l_{s}\right)$, which gives:

$$
\tan \left(\beta l_{A}\right)=-\frac{2 \tan \left(\beta \Delta l_{s}\right)}{1-\tan ^{2}\left(\beta \Delta l_{s}\right)} .
$$

where $l_{A}$ is the length of the loop structure. Using the properties of the tangent function gives:

$$
\tan \left(\beta l_{A}\right)=-\tan \left(2 \beta \Delta l_{S}\right),
$$

which is solved by:

$$
l_{A}+2 \Delta l_{s}=m \lambda_{r} / 2,
$$

where $m$ is an integer.

For the C-like scatterer, after inserting (1) and (2) into (15), we have:

$$
\tan \left(\beta l_{B}\right)=\frac{1-\tan \left(\beta \Delta l_{s}\right) \cdot \tan \left(\beta \Delta l_{o}\right)}{\tan \left(\beta \Delta l_{s}\right)+\tan \left(\beta \Delta l_{o}\right)} .
$$

where $l_{B}$ is the length of the C-like scatterer. Once again, the use of the properties of the tangent function gives:

$$
\begin{aligned}
\tan \left(\beta l_{B}\right) & =1 / \tan \left[\beta\left(\Delta l_{s}+\Delta l_{o}\right)\right] \\
& =\tan \left[\pi / 2-\beta\left(\Delta l_{s}+\Delta l_{o}\right)\right],
\end{aligned}
$$

which is solved by:

$$
l_{B}+\Delta l_{s}+\Delta l_{o}=\lambda_{r} / 4+m \lambda_{r} / 2 .
$$

where $m$ is an integer. 


\section{REFERENCES}

[1] K. C. Gupta, R. Garg, I. Bahl, and P. Bhartia, 'Coplanar lines: Coplanar waveguide and coplanar strip', in Microstrip Lines and Slotlines 2nd Ed., 2 edition., Artech House Publishers, 1996.

[2] Y.-H. Suh and K. Chang, 'Coplanar stripline resonators modeling and applications to filters', IEEE Trans. Microw. Theory Tech., vol. 50, no. 5, pp. 1289-1296, May 2002.

[3] N. Yang, C. Caloz, and K. Wu, 'Fixed-Beam Frequency-Tunable Phase-Reversal Coplanar Stripline Antenna Array', IEEE Trans. Antennas Propag., vol. 57, no. 3, pp. 671-681, Mar. 2009.

[4] A. Vena, E. Perret, and S. Tedjini, 'Design of Compact and AutoCompensated Single-Layer Chipless RFID Tag', IEEE Trans. Microw. Theory Tech., vol. 60, no. 9, pp. 2913-2924, Sep. 2012.

[5] Y. Ning, C. Multari, X. Luo, C. Palego, D. Molinero, X. Cheng, J. C. M. Hwang, and C. Merla, 'Coplanar stripline microchamber for electrical detection of live and dead biological cells', in Microwave Conference (EuMC), 2013 European, 2013, pp. 475-478.

[6] R. N. Simons, N. I. Dib, and L. P. B. Katehi, 'Modeling of coplanar stripline discontinuities', IEEE Trans. Microw. Theory Tech., vol. 44, no. 5, pp. 711-716, May 1996.

[7] L. Zhu and K. Wu, 'Field-extracted lumped-element models of coplanar stripline circuits and discontinuities for accurate radiofrequency design and optimization', IEEE Trans. Microw. Theory Tech., vol. 50, no. 4, pp. 1207-1215, Apr. 2002.

[8] W. J. Getsinger, 'End-effects in quasi-TEM transmission lines', IEEE Trans. Microw. Theory Tech., vol. 41, no. 4, pp. 666-672, Apr. 1993.

[9] C.-W. Chill, 'Equivalent circuit parameters of coplanar stripline discontinuities', Microw. Antennas Propag. IEE Proc., vol. 149, no. 1, pp. 11-16, Feb. 2002.

[10] T. C. Edwards, M. B. Steer, T. C. Edwards, and M. B. Steer, 'Discontinuities in Microstrip and Stripline', in Foundations of Interconnect and Microstrip Design, John Wiley \& Sons, Ltd, 2000, pp. 225-268.

[11] B. Easter, 'The Equivalent Circuit of Some Microstrip Discontinuities', IEEE Trans. Microw. Theory Tech., vol. 23, no. 8, pp. $655-660$, Aug. 1975 .

[12] S. Bories, M. Hachemi, K. H. Khlifa, and C. Delaveaud, 'Small antennas impedance and gain characterization using backscattering measurements', in 2010 Proceedings of the Fourth European Conference on Antennas and Propagation (EuCAP), 2010, pp. 1-5.

[13] W. Wiesbeck and E. Heidrich, 'Wide-band multiport antenna characterization by polarimetric RCS measurements', IEEE Trans. Antennas Propag., vol. 46, no. 3, pp. 341-350, Mar. 1998.

[14] O. Vicencio and J. Romeu, 'Application of RCS measurement method for characterization of small antennas', in IEEE Antennas and Propagation Society International Symposium, 2008. AP-S 2008, 2008, pp. 1-4.

[15] E. Perret, Radio Frequency Identification and Sensors: From RFID to Chipless RFID. John Wiley \& Sons, 2014.

[16] G. Ghione and C. Naldi, 'Analytical formulas for coplanar lines in hybrid and monolithic MICs', Electron. Lett., vol. 20, no. 4, pp. 179181, Feb. 1984.

[17] O. Rance, R. Siragusa, P. Lemaître-Auger, and E. Perret, 'Toward RCS Magnitude Level Coding for Chipless RFID', IEEE Trans. Microw. Theory Tech., vol. 64, no. 7, pp. 2315-2325, Jul. 2016.

[18] C. E. Baum, E. J. Rothwell, K. M. Chen, and D. P. Nyquist, 'The singularity expansion method and its application to target identification', Proc. IEEE, vol. 79, no. 10, pp. 1481-1492, Oct. 1991.

[19] Chipless RFID - Design Procedure and Detection Techniques | Reza Rezaiesarlak | Springer.

[20] A. Ramos, E. Perret, O. Rance, S. Tedjini, A. Lázaro, and D. Girbau, 'Temporal Separation Detection for Chipless Depolarizing FrequencyCoded RFID', IEEE Trans. Microw. Theory Tech., vol. 64, no. 7, pp. 2326-2337, Jul. 2016.

[21] W. Wiesbeck and D. Kahny, 'Single reference, three target calibration and error correction for monostatic, polarimetric free space measurements', Proc. IEEE, vol. 79, no. 10, pp. 1551-1558, Oct. 1991.

[22] A. Vena, E. Perret, and S. Tedjni, 'A Depolarizing Chipless RFID Tag for Robust Detection and Its FCC Compliant UWB Reading System', IEEE Trans. Microw. Theory Tech., vol. 61, no. 8, pp. 2982-2994, Aug. 2013.

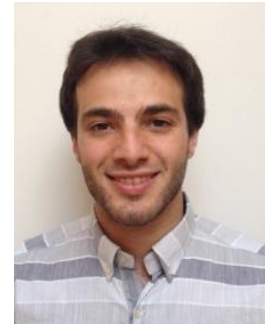

Olivier Rance (S'15) is a PhD student working under the supervision of Dr. Etienne Perret. He received his Master's degree in Electronic Engineering from the Institute National Polytechnique de Grenoble (Grenoble-INP), and joined the LCIS in 2012. His main research interests are leaky-wave antennas and chipless RFID.

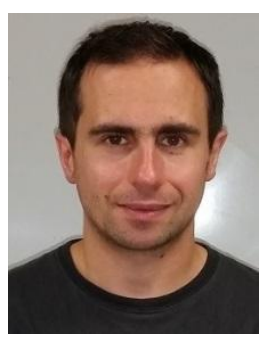

Romain Siragusa was born in Paris, France. He received the engineer degree and the master degree from the Institute National Polytechnique de Grenoble (Grenoble-INP) in 2006 and the Ph.D degree from the same institute at the Laboratoire de Conception et d'Intégration des Systèmes (LCIS) in Valence, France. In 2010/2009, he joined the "commissariat à l'énergie atomique" (CEA) as post doctoral fellow where he studied the high impedance surface for integrated antenna on silicon. Since 2011, he is associate professor at Grenoble-INP Esisar / LCIS. His current scientific interests include leakywave structures and Chipless RFID.

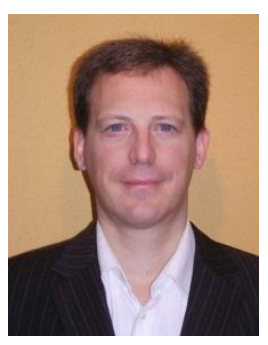

Pierre Lemaître-Auger received the B. Ing. and the M. Sc. A. degrees from the École Polytechnique de Montréal, Canada, in 1992 and 1994 respectively in physical engineering. He obtained his doctor degree in Optoelectronics and Microwave from the Institut National Polytechnique de Grenoble, France, in 1998. He joined the engineering school Esisar (Valence, France) of the Institut Polytechnique de Grenoble and the LCIS Laboratory in 1998 as an associate professor $\mathrm{He}$ participated to the creation of the Esisar and especially to the elaboration of its physics teaching program. He directed the Physical Department of the Esisar from 1997 to 2006. From 2006 to 2009, he was the Director of Study of that same school. In 2010-2011, he was visiting professor at the École Polytechnique de Montréal in the Poly-Grames Microwave Research Center. Since 2014, he is Head of International Relations of Esisar. His first research topics concerned integrated optics on glass substrates for sensors applications. From 2004 to 2010, he actively participated to the creation of a French startup company specialized in optical sensors. In 2006, he changed his research activities to electromagnetic wave generation and propagation: antennas, array antennas, frequency selective surface and localized waves. He is the author and co-author of more than 80 journal papers, letters, and international conferences. He is co-author of 5 patents. His work on wall-paper blocking WiFi signals won several French awards. 


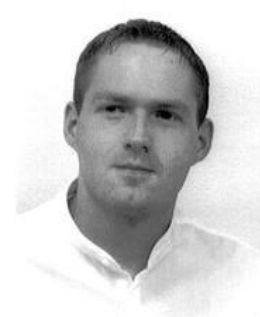

Etienne Perret received the Eng. Dipl. in electrical engineering from the Ecole Nationale Supérieure d'Electronique, d'Electrotechnique, d'Informatique, d'Hydraulique, et des Télécommunications, Toulouse, France, in 2002, and the M.Sc. and Ph.D. degrees from the Toulouse Institute of Technology, France, in 2002 and 2005, respectively, all in electrical engineering. From 2005 to 2006, he held a post-doc position at the Institute of Fundamental Electronics (IEF), Orsay, France. Since September 2006, Dr. Perret is Associate Professor in electrical engineering with the Grenoble Institute of Technology. In October 2014, he was elevated to Junior member of the "Institut Universitaire de France", an institution that distinguishes professors for their research excellence, as evidenced by their international recognition.

$\mathrm{He}$ is authored and co-authored of more than 120 technical conferences, letters and journal papers, and books. He is IEEE senior member and Technical Program Committee member of the IEEE International Conference on RFID. He was keynote speaker and the chairman of several international symposiums. His research activities cover the electromagnetic modeling of passive devices for millimeter and submillimeter-wave applications. His current research interests are in the field of wireless communications, especially radio frequency identification (RFID) and chipless RFID. His interests also involve advanced computer aided design techniques based on the development of an automated co-design synthesis computational approach. He was named one of MIT Technology Review's French Innovator's under 35 in 2013 for his work on chipless RFID. He is also the recipient of the French's Innovative techniques for the environment awards in 2013. 